\title{
SAÚdE COLETIVA E ATIVIDAdE FÍSICA: AS POLÍTICAS PÚBLICAS RESPONDEM E INCORPORAM AS REALIDADES SETORIAIS?
}

\author{
Ana Paula Martins Vicentin \\ Aguinaldo Gonçalves
}

\section{Resumo}

Assevera-se na identidade da Promoção da Saúde, o significado dos chamados Determinantes Sociais da Saúde, tanto a nível internacional, quanto no âmbito nacional, com destaque para as implicações da atuação da respectiva Comissão Nacional. Nesse quadro teórico-metodológico, situa-se a Saúde Coletiva e Atividade Física como área de conhecimento e intervenção, comprometida com a redução da morbimortalidade devida ao sedentarismo e hipocinesia, tomando-se diferentes projetos realizados em nosso meio como referências de factibilidade e pertinência. Contemplam-se aí ações desenvolvidas com Agentes Comunitários de Saúde, bem como programas para o controle da obesidade e hipertensão arterial, os quais a partir de necessidades biológicas básicas, incorporaram aspectos sociais relevantes, como organização popular, transporte digno, equipamentos para esporte e lazer, reciclagem de materiais utilizados, superação de condições inadequadas de moradia, violência urbana e ausência do poder público. A questão que se coloca na direção das duas ordens de fato apresentadas é em que medida as políticas públicas setoriais vigentes em nosso país poderão ampliar iniciativas dessa natureza, fazendo-as migrar do interior do pioneirismo acadêmico para a realidade do coletivo.

\section{Palavras-Chave}

Promoção da saúde; Atividade física; Políticas públicas

\section{PHYSICAL ACTIVITY AND PUBLIC HEALTH: PUBLIC POLICIES RESPOND AND INCORPORATE THE REALITIES SECTOR?}

\author{
Ana Paula Martins Vicentin \\ Aguinaldo Gonçalves
}

\begin{abstract}
States in the identity of the Promotion of Health, the meaning of the Social Determinants of Health, both at the international and the national level, with emphasis on the implications of the actions of their National Commission. In this theoretical and methodological view, situates the Public Health and Physical Activity as an area of knowledge and action, committed to the reduction of morbidity due to inactivity and hypokinesia, taking up various projects in our country as a reference for feasibility and relevance. They include actions developed with Community Health Agents, as well as programs to control obesity and hypertension, which from basic biological needs, incorporate relevant social and popular organization, transport, decent equipment for sports and leisure, recycling of materials used, overcoming inadequate housing, urban violence and lack of public power. The question that arises in the direction of two orders of fact presented is the extent to which sectoral policies in force in our country may expand such initiatives, making them migrate from within the academic pioneers in the reality of the collective.
\end{abstract}

Conexões: revista da Faculdade de Educação Física da UNICAMP, Campinas, v. 7, n. 2, p. 24-37, maio/ago. 2009. 


\section{Key-Words}

Health promotion; Physical activity, Public policy 


\section{INTRODUÇÃO}

O campo da Promoção da Saúde (PS) vem desde o início do século XX trazendo novos modelos conceituais e aplicados. Surgiu como reação à medicalização da sociedade (SIGERIST, 1946), baseada na articulação de saberes técnicos e populares pela mobilização de recursos institucionais comunitários, públicos e privados para o enfrentamento e resolução das questões em saúde (BUSS, 2003) .

Paralelamente ao referido panorama internacional, na América Latina, mais especificamente no Brasil, setores e profissionais atentos às características locais e comprometidos com as liberdades democráticas (GONÇALVES, 2004) protagonizaram a Reforma Sanitária Brasileira, que alçou conquistas significativas. Tal processo foi gerador do que se conhece atualmente por Saúde Coletiva (SC), ou o recorte do conhecimento e da intervenção humana que lida com a relação saúde-doença-cuidado no âmbito singular dos indivíduos e plural dos grupos (CORRÊA FILHO et al., 1994).

Alguns acontecimentos como a Constituição Cidadã de 1988 e a lei 8080/1990 - que se refere à criação do Sistema Único de Saúde (SUS) - consagram esse ideário apontando saúde como direito do ser humano e dever do Estado. Assim, o movimento internacional constituído pela Nova PS e pela SC, fruto das discussões e avanços dos países latino-americanos, vem enfatizar questões de grande valia não só para o acesso universal e igualitário aos serviços, mas também às políticas que visem à redução de doenças e outros agravos.

O texto presente detalha como se deu o processo acima, na direção da consolidação e aplicação institucionais da Promoção à Saúde, para, a seguir, situar, em seu interior, necessidades e ações precursoras da Atividade Física face aos agravos da contemporaneidade sedentária e hipocinética.

\section{PROMOÇÃO DA SAÚDE E OS DETERMINANTES SOCIAIS EM SAÚDE}

No Canadá em 1974, Marc Lalonde, então ministro da Saúde, deu início a profunda mudança nas concepções e práticas do setor saúde a nível internacional ao formular a proposição da saúde baseada na biologia humana, meio ambiente, estilo de vida e organização da atenção à saúde, fundamentada nas atuações do governo do seu país. Tais preceitos deram início a grande ênfase ao estilo de vida, delegando ao indivíduo a responsabilidade de geração ou não de dano à saúde. Em 1976 a Organização Mundial da Saúde (OMS) apresentou a estratégia global Saúde para Todos no Ano 2000, visando a extensão da 
cobertura dos serviços básicos com sistemas de assistência, além de já trazer também a idéia de combate aos determinantes sociais; no entanto, o contexto vivido não se configurou favorável e as recomendações elaboradas converteram-se em ações eficazes em poucas ocasiões.

Diante dessas consignas foram conquistados muitos avanços na assistência a saúde, porém a ênfase no estilo de vida estimulou a perspectiva crítica, dado o que ficou conhecido como culpabilização da vítima, isto é, o entendimento dos processos pelos quais determinados grupos, à margem do desenvolvimento capitalista, passam a ser responsabilizados por problemas de saúde cujas causas se encontram fora de sua governabilidade (LABONTE, 1993). Nesse caso, o grande vilão seria o próprio cidadão, a depender, dentro do contexto sócio-político da sua existência, de decisões pessoais próprias, sem considerar as relações de poder e limitação de acesso (GONÇALVES, 2005).

A participação de instituições como a OMS e a Organização Panamericana de Saúde (OPAS) valoriza outros requisitos, como a justiça social, educação, saneamento e habitação. De maneira simplificada, apontou-se para necessidade de tecnologia adequada, crítica ao elitismo médico e a forte conexão entre a saúde e o social, chamada de atenção primária abrangente. O documento marco da Nova Promoção da Saúde é a Carta de Ottawa, fruto da I Conferência realizada no Canadá, que define PS como "processo de capacitação da comunidade para atuar na melhoria da sua qualidade de vida e saúde, incluindo uma maior participação no controle desse processo" (OPAS, 2006a).

$\mathrm{Na}$ ocasião, foram consensados cinco campos de ação: política pública saudável, ambientes favoráveis, reforço da ação comunitária, desenvolvimento de habilidades das pessoas e reorientação dos serviços de saúde. O primeiro deles pode ser caracterizado pelo interesse e preocupação explícitos de todas as áreas em relação à saúde e à equidade e pelos compromissos de tais impactos sobre a população (OPAS, 2006b). Além disso, tem-se como meta o apoio à saúde da mulher, à alimentação, à nutrição e ao combate do tabaco e álcool. Declarou-se o ambiente favorável de extrema importância, e como meios para sua promoção e criação, medidas de i) mobilização comunitária; ii) capacitação de indivíduos e comunidade a partir da educação e participação nos processos de tomada de decisão; iii) construção de alianças com objetivos comuns; e iv) mediação de interesses conflitantes na sociedade, para assegurar o acesso igualitário em saúde (OPAS, 2006c). 
Buscando incorporar principalmente o questionamento das ações de prevenção e controle das doenças considerando as relações sociais de exploração (CARVALHO, 2005), a Saúde Coletiva, entre nós, desde pronto, vem revelando, nesse contexto, i) a saúde como resultante de múltiplos determinantes sociais, e ii) a temática do trabalho em saúde. Com objetivo de esclarecer quais as contribuições trazidas pela SC (PAIN; ALMEIDA FILHO, 1998), apontou-se que, enquanto campo do saber, trabalha ela com o estudo do fenômeno saúde/doença em populações e enquanto ente social investiga a produção e distribuição das doenças na sociedade e também procura compreender, enfim, as formas com que esta identifica suas necessidades e problemas de saúde, e como maneja explicação e organização para enfrentá-los.

Mais recentemente tem se destacado a premência de compreender a influência de fenômenos atuais como a globalização e os meios de comunicação de massa (OPAS, 2006d) para as ações em PS, reforçando a abordagem dos determinantes econômicos, ambientais e o fortalecimento dos mecanismos de colaboração da sociedade (OPAS, 2006e). Em 2004, o então diretor geral da OMS, Lee Jong-Wook propôs na Assembléia Mundial, a criação de grupo com o mérito de indicar políticas na área (OMS, 2006). Nomeou-se efetivamente a Comissão de Determinantes Sociais em Saúde (CDSS - OMS) em março de 2005, com atuação de levar conhecimento à ação ou, mais claramente, utilizar evidências científicas como alavanca transformadora.

Estruturalmente, a CDSS-OMS contém três linhas de trabalho:

- sistematização de evidências sobre experiências e formulação de medidas;

- fomento ao debate junto à sociedade;

- definição de compromissos de médio e longo prazo com vistas a incorporar as discussões sobre as desigualdades em saúde.

Os representantes da Comissão inicialmente se debruçaram sobre como a saúde tem sido historicamente, produzindo o documento "Ação sobre os determinantes sociais da saúde: aprendendo com experiências anteriores". De maneira oportuna, apresentaram-se áreas com questões específicas. Primeiramente, percebeu-se a definição de quão ambiciosas serão as metas. Citam-se experiências já vivenciadas em fins da década de 70, com a atenção primária abrangente e seletiva. Aspectos mais superficiais também foram pensados, como os meios de comunicação e respectivas implicações. Em suma, a comissão definiu a identidade de sua atuação, e para isso sistematizou quatro níveis, ou seja, contextos em que as intervenções podem se dar, como, com a redução de:

Conexões: revista da Faculdade de Educação Física da UNICAMP, Campinas, v. 7, n. 2, p. 24-37, maio/ago. 2009. 
- estratificação social, alterando a hierarquia socioeconômica através de medidas de redistribuição;

- exposição por parte de grupos em posição menos privilegiada a fatores prejudiciais;

- vulnerabilidade de populações carentes às condições danosas;

- conseqüência desigual dos problemas de saúde e deterioração socioeconômica de indivíduos que adoecem.

A comissão, a seguir, situa que os motivos de insucesso já existentes podem ser aplicações inadequadas ou retaliações político-econômicas em função do poder e do lucro. Adiantando-se às repercussões que a CDSS - OMS poderia causar, foram identificados os possíveis opositores: a classe médica, o governo, setores corporativos e organizações financeiras, e em como agir contra obstáculos por eles gerados. A Comissão indigitou redes de alianças com atores de vários níveis, incluindo representantes globais e governos, o setor comercial e as organizações da sociedade civil, no âmbito internacional e nacional.

Outra preocupação dos especialistas voltou-se ao discurso utilizado para disseminar seus princípios, reforçando a necessidade de amplas evidências para a formulação desses. Aproximar o comprovado cientificamente de linguagem agradável que indique claramente possibilidades de realização foi considerado dos maiores desafios da CDSS - OMS.

Em julho de 2005, a OPAS reuniu em Washington os países da América e apresentou as propostas da CDSS - OMS. Após esse encontro houve, no Brasil, grande interesse de diversas instituições, destacandose a Fundação Oswaldo Cruz (Fiocruz) e o Sistema de Vigilância em Saúde do Ministério da Saúde que lideraram o processo de agenda de atividades buscando respostas para o enfrentamento dos DSS locais. Como o primeiro país a responder ao chamado da OMS, foi lançada em março de 2006, a Comissão Nacional sobre Determinantes Sociais em Saúde (CNDSS), constituída pelo então Ministro da Saúde e mais dezessete integrantes, com diversas trajetórias profissionais - entre sanitaristas, juristas, cantores e cartunistas - e coordenada pelo presidente da Fiocruz que, na ocasião, ressaltou a importância histórica dessa criação no momento de comemoração dos vinte anos do SUS.

Dentre os objetivos da Comissão Nacional relevam-se: estimular a melhoria da qualidade e a completude das informações sócio-demográficas nos sistemas oficiais da saúde, para permitir o monitoramento dos DSS; introduzir a temática e suas conseqüências na formação profissional; mobilizar a sociedade civil 
para a defesa do princípio da equidade na execução das políticas públicas pertinentes. A seguir, reconheceu ela a desigualdade injusta e evitável - ou seja, a iniqüidade - como a mais grave doença do país. Com execução em dois anos, seus constituintes tiveram a meta de agir o quanto antes, sendo o ponto de partida os conhecimentos já produzidos no Brasil (BRASIL, 2006). Para se poder atinar com o alcance de tais perspectivas, cabe entender a identidade dos DSS: trata-se das condições em que as pessoas vivem e trabalham, ou "são as característica sociais, dentro das quais a vida transcorre" (TARLOV, 1996). Essa concepção veio em congruência com outras formas de tratar saúde, buscando atuar nos fatores que ajudam as pessoas a ficarem saudáveis, ao invés do auxílio que obterão quando se tornarem doentes: ressaltando a necessidade de mudança de prioridade, situa-se como eixo fundamental: "que se considere a importância da equidade, de modo que o desenvolvimento humano - e não só o financeiro - esteja no centro da política econômica" (PELLEGRINI FILHO, 2006).

Já em agosto de 2008, o ex-ministro Adib Jatene foi encarregado de apresentar o relatório final da Comissão, intitulado As causas sociais das iniqüidades em saúde no Brasil: vincou o compromisso com a equidade, a ação e evidencias científicas que justifiquem e fundamentem as afirmações do documento.

Como fruto do processo, é enfatizada a criação da Secretaria de Atenção Primária e Promoção da Saúde, com a finalidade de levar a permanente discussão sobre o tema à agenda governamental. São apontados fatos que marcaram a história social e da saúde brasileira, como a urbanização desenfreada, a recente inversão da pirâmide etária brasileira, o aumento do Produto Interno Bruto, e o não acompanhamento da melhoria na distribuição de renda, bem como a maneira em que todos esses acontecimentos influenciam a saúde dos diversos grupos populacionais no país. O relatório registra também avanços significativos, como a menor diferença na expectativa de vida se comparadas as regiões sul e nordeste (a diferença que em 1960 era de 20 foi para 5 anos em 1996) e a diminuição da mortalidade infantil.

Em segmento específico sobre "Comportamentos, estilos de vida e saúde" são lembrados tabagismo, alcoolismo e sedentarismo e maneiras de combatê-los, como regulação de propaganda e o cumprimento da proibição de comercialização de bebidas para menores, bem como suporte e educação preventiva.

Além de retratar a situação de saúde, o texto traz recomendações sobre a ação na busca por maior equidade como a participação social e o empoderamento de grupos mais vulneráveis. Destaca também a 
$\overline{\text { necessidade de articulação entre os diferentes âmbitos governamentais, a partir da análise de políticas }}$ e programas em andamento relacionados aos determinantes.

\section{SAÚDE COLETIVA E ATIVIDADE FÍSICA}

Compativelmente com toda essa realidade macro-social e política, nestes últimos anos pode-se observar o aumento da visibilidade que os organismos internacionais têm dado para a questão da atividade física (AF). Em 2003, ela foi identificada como prioridade para saúde pública em documento da OMS (WHO, 2003), face à sua importante relação com causas de mortalidade (WHO, 2007). No mesmo ano publicouse o relatório denominado "Doenças crônico-degenerativas e obesidade: estratégia mundial sobre alimentação saudável, atividade física e saúde” (OPAS, 2003). Tal referência indica ao nível local, nacional e internacional o desenvolvimento de atividades para redução dos fatores de risco e das taxas de morbidade e mortalidade da população acometida por enfermidades crônicas, associadas com o regime alimentar e sedentarismo. Ainda assim, o programa "Mais Saúde" também enumera entre seus objetivos o estímulo a hábitos saudáveis e, entre eles a prática de AF (BRASIL, 2007).

De fato, estudos realizados em países como México, Peru e Brasil mostram que o hábito de exercícios regulares é pouco freqüente e está relacionado ao maior nível educacional e acesso a bens e produtos, ficando, de modo geral, mais distante para aqueles que têm menor renda (SECLÉN-PALANCÍN; JACOBY, 2003; HERNÁNDEZ et al., 2003; MONTEIRO et al., 2003). No Brasil, o que o Programa Nacional de Promoção da AF propõe é a veiculação das recomendações do Center for Disease Control of Sports Medicine e American College of Sports Medicine, ou seja, trinta minutos de atividade física por dia na maior parte dos dias da semana, se possível todos, de forma contínua ou acumulada e abrangendo três ambientes básicos: atividades domésticas, transporte e atividades de lazer (PATE et al., 2007).

Em nosso meio, o Grupo de Saúde Coletiva Epidemiologia e Atividade Física (GSCEAF) tem operado a respeito, no referente a especificidades. Assim é que inseriu conteúdos da AF no dia a dia dos Agentes Comunitários de Saúde em projeto do Jardim Santa Mônica, Campinas (MOURA et al., 2002). A tentativa de aproximar a população à AF consistiu inicialmente do conhecimento da rede de serviços locais de saúde e da identidade de procedimentos para operações intra e extra muros na Unidade do SUS. Conclusivamente, além de os agentes de saúde conseguirem introjetar em seu agir profissional a contribuição da AF nas práticas sociais, foi explicitada também a percepção de que a forma com que cada um vive seu quotidiano não é opção essencialmente pessoal. 
Depois da aproximação inicial, e com o foco em grupo de clientes, aplicou-se contribuição ao manejo da hipertensão arterial, na mesma Unidade de saúde. Após interação com interessados, havia orientação ou supervisão do professor de Educação Física, que atuava em conjunto com equipe médica, promovendo três sessões semanais de AF, com intensidade e freqüência controlada (CAMPANE et al., 2002). Além da consecução de seus objetivos intrínsecos, a iniciativa rendeu proximidade com a equipe de saúde local, o que possibilitou outras realizações na região.

Ampliando a abordagem, no caso em direção à crescente problemática da obesidade nos tempos atuais, desenvolveu-se trabalho com objetivo de verificar alterações na classificação do Índice de Massa Corporal e Índice Cintura Quadril de obesos, com programa de AF que incluía várias opções aeróbias, com ênfase em caminhadas e hidroginástica. Partindo de dados disponíveis em prontuários, foram observados indivíduos com idades entre 40 e 45 anos. Comprovou-se redução de massa corpórea em 55\% dos homens e em 75\% das mulheres, além da diminuição da relação cintura-quadril em 42,5\% do público masculino e $72,5 \%$ do feminino (CARVALHO et al., 2003). Com base nas observações, os autores concluíram que foi possível promover diminuição dos índices antropométricos e conseqüentemente nos níveis de risco à saúde dos pacientes (CARVALHO et al., 2002). Ademais, tais estudos viabilizaram competência específica no tratamento com referido público, considerando suas características e peculiaridades.

Ainda na intencionalidade de gerar contribuições acerca da relação da $\mathrm{AF}$ e excesso de peso, em intervenção executada com atividade aquática, atuaram com obesas para verificar melhora das variáveis biomotoras e composição corporal. Durante 17 semanas, houve três sessões semanais de 50 minutos de duração e avaliações inicial e final. Ao final, detectaram-se ganhos de manutenção do peso, melhora da flexibilidade e da força, redução da gordura corporal e aumento da condição cardiorrespiratória (PASETTI et al., 2004). Essa linha de atuação continua em vigência com publicações registradas no site do GSCAEF (www.unicamp.br/fef).

Em outra frente, houve a iniciativa com mulheres sedentárias com idades entre 35 e 45 anos, moradoras da região do São Marcos. Elegeu-se como variável independente a participação em programa de hidroginástica e como dependentes peso, índice de massa corporal, flexibilidade, imagem corporal, e índice de qualidade de vida. Foram realizadas três sessões semanais, com duração de 60 minutos, durante treze semanas, e testes controles antes e depois. Além dos resultados adaptativos gerados, com destaque 
para ganho significativo de flexibilidade, acrescenta-se a realização de gestões não só individuais que conduziram a conquistas nos padrões dos agires e fazeres (GONÇALVES; VICENTIN, 2004), como fontes coletivas de recurso financeiro, obtenção de transporte e equipamentos de lazer e esportes, além de vestimentas adequadas à prática em tela. Após as discussões, propiciaram-se soluções internas e respectivas mobilizações. A ação foi continuada com a auto-organização, como a adoção de rifas, venda de artigos, inserção no processo de coleta e reciclagem de materiais e execução de trabalhos manuais.

\section{CONSIDERAÇÕES FINAIS}

Pela apresentação das experiências pioneiras de conhecimento e atuação sobre necessidades populacionais de Atividade Física, fiéis às respectivas coordenadas dos organismos nacionais e internacionais da Saúde, foi possível observar que iniciativas específicas conduzem ao envolvimento com situações sociais mais amplas, como o SUS, violência urbana, condições de moradia inadequadas e ausência da administração pública. Dessa forma, surgidas situações na execução dos projetos levando à interlocução, tanto dos organizadores quanto dos participantes, com organizações não governamentais, representantes parlamentares, empresas privadas e autoridades estatais, reafirmaram exatamente a valorização e incentivo de propostas atuais da OMS como o empowerment e o advocacy.

Postos estes termos, importa explorar como dificuldades globais, geradas propriamente por políticas econômicas que consideram o capital e o comércio e desconsideram os excluídos, em panorama marcado pela desigualdade social e iniqüidades, possam ser superadas por gestões que expandam as acumulações setorialmente conquistadas, fazendo-as migrar do interior do pioneirismo acadêmico para a realidade do coletivo.

\section{REFERÊNCIAS}

BRASIL. Ministério da Saúde. Programa de Aceleração do Crescimento da Saúde. Programa Mais Saúde: 2007. Disponível em: <http://bvsms.saude.gov.br/bvs/pacsaude/index.php>. Acesso em: 05 dez. 2007.

BRASIL. Comissão Nacional de Determinantes em Saúde. Grupo Eclético, Missão Comum. RADIS, v. 45, p. 23, 2006.

BUSS, P. M. Uma introdução ao conceito de promoção da Saúde. In: CZERESNIA, D.; FREITAS, C. M. (Org.). Promoção da saúde: conceitos, reflexões, tendências. Rio de Janeiro: Fiocruz, 2003. 
CAMPANE, R. Z.; GONÇALVES, A.; CORREA, C. R. A Atividade Física no controle de hipertensão arterial nos Serviços Básicos de Saúde, a partir do Projeto São Marcos, Campinas, SP. In: SIMPÓSIO DE EDUCAÇÃO FÍSICA E DESPORTOS DO SUL DO BRASIL, 14., Ponta Grossa, 2002. Anais... Ponta Grossa, 2002.

CARVALHO, S. R. Saúde coletiva e promoção da saúde: sujeito e mudança. São Paulo: Hucitec, 2005. CARVALHO, P. J. A. et al. Efeitos adaptativos em índices antropométricos, decorrentes de programa de Exercícios Físicos associados à dieta, em obesos internados em SPA médico. In: SIMPÓSIO INTERNACIONAL DE CIÊNCIAS DO ESPORTE, 25., São Paulo, out. 2002. Anais... São Paulo, 2002. CARVALHO, P. J. A.; PADOVANI, C. R.; GONÇALVES, A. Evolução na classificação de índices antropométricos, relacionados a fatores de risco à saúde, no tratamento da obesidade em spa médico. In: CONGRESSO INTERNACIONAL DE EDUCAÇÃO FÍSICA E MOTRICIDADE HUMANA, 3., Rio Claro, 2003. Anais... Rio Claro, 2003.

CORRÊA FILHO, H. R.; GONÇALVES, N. N. S.; GONÇALVES, A. Ciência e tecnologia em saúde no Brasil: modelo de identificação de prioridades setoriais. Rev Bras Admin Publica, São Paulo, v. 28, n.4 p. 18-26, 1994.

GONÇALVES, A. Agentes comunitários de saúde: choque de povo. Cad Saúde Pública, Rio de Janeiro, v. 20, n. 6, p. 1771-1772, 2004.

. Qualidade de vida. In: GONZÁLEZ, F. J.; FENSTERSEIFER, P. E. (Org.). Dicionário crítico de Educação Física. Ijuí: Ed. da Unijuí, 2005. p. 354-356.

.; VICENTIN, A. P. M. Promovendo e exercendo Economia Solidária através da Saúde e do Esporte: a experiência da Unicamp no Jardim São Marcos, Campinas, SP. In: ENCONTRO INTERNACIONAL DE ECONOMIA SOLIDÁRIA, 2., São Paulo, 2004. Anais... São Paulo, 2004.

HERNÁNDEZ, B.; HAENE, J.; BARQUERA, S. et al. Factores asociados con la actividad física en mujeres mexicanas en edade reproductiva. Rev Panan Salud Publica, v. 14, n. 4, p. 235-245, 2003.

LABONTE, R. Health promotion and empowerment: practice frameworks. Toronto: Center for Health Promotion, 1993.

MONTEIRO, C. A. et al. A descriptive epidemiology of leisure-time physical activity in Brazil, 19961997. Rev Panam Salud Publica, v. 14 n.4 p. 246-254, 2003.

MOURA, A. C. S.; GONÇALVES, A.; CORRÊA C. R. S. Atividade física na capacitação de agentes comunitários: a experiência na Unidade Básica de Saúde (UBS) do Jardim Santa Mônica - Campinas-SP. In: FÓRUM BRASILEIRO DE EDUCAÇÃO FÍSICA E CIÊNCIAS DO ESPORTE, Viçosa, 2002. Anais... Viçosa, 2002.

Conexões: revista da Faculdade de Educação Física da UNICAMP, Campinas, v. 7, n. 2, p. 24-37, maio/ago. 2009. ISSN: 1983-930. 
ORGANIZAÇÃO MUNDIAL DE SAUDE (OMS). Ação sobre os determinantes sociais da saúde: aprendendo com experiências anteriores. Secretaria da Comissão sobre Determinantes Sociais da Saúde, março de 2005. Disponível em: <http://www.determinantes.fiocruz.br/pdf/texto/T4-1_MarmottAÇÃO\%.pdf>. Acesso em: 29 dez. 2006.

ORGANIZAÇÃO PANAMERICANA DE SAUDE (OPAS). Doenças crônico-degenerativas $e$ obesidade: estratégia mundial sobre alimentação saudável, atividade física e saúde. Brasília: Organização Pan-Americana de Saúde, 2003.

ORGANIZAÇÃO PANAMERICANA DE SAUDE (OPAS). CONFERÊNCIA INTERNACIONAL SOBRE PROMOÇÃO DA SAÚDE, 1., Ottawa, 1986. Anais... Ottawa, 1986. Disponível em: $<$ http://www.opas.org.br/coletiva/uploadArq/Ottawa.pdf $>$. Acesso em: 02 out. 2006a.

ORGANIZAÇÃO PANAMERICANA DE SAUDE (OPAS). CONFERÊNCIA INTERNACIONAL SOBRE PROMOÇÃO DA SAÚDE, 2., Adelaide, 1988. Anais... Adelaide, 1988. Disponível em: $<$ http://www.opas.org.br/coletiva/uploadArq/Adelaide.pdf $>$. Acesso em: 02 out. 2006b.

ORGANIZAÇÃO PANAMERICANA DE SAUDE (OPAS). CONFERÊNCIA INTERNACIONAL SOBRE PROMOÇÃO DA SAÚDE, 3., Sundsvall, 1991. Anais... Sundsvall, 1991. Disponível em: $<$ http://www.opas.org.br/coletiva/uploadArq/Sundsvall.pdf $>$. Acesso em: 02 out. 2006c.

ORGANIZAÇÃO PANAMERICANA DE SAUDE (OPAS). CONFERÊNCIA INTERNACIONAL SOBRE PROMOÇÃO DA SAÚDE, 4., Jacarta, 1997. Anais... Jacarta, 1997. Disponível em: $<$ http://www.opas.org.br/coletiva/uploadArq/Jacarta.pdf $>$. Acesso em: 02 out. 2006d.

ORGANIZAÇÃO PANAMERICANA DE SAUDE (OPAS). CONFERÊNCIA INTERNACIONAL SOBRE PROMOÇÃO DA SAÚDE, 5., México, 2000. Anais... México, 2000. Disponível em: $<$ http://www.opas.org.br/coletiva/uploadArq/Mexico.pdf $>$. Acesso em: 02 out. 2006e.

PAIM, J. S.; ALMEIDA FILHO, N. Saúde coletiva: uma "nova saúde pública" ou campo aberto a novos paradigmas? Rev Saúde Pública, v. 32 n. 4, p. 299-316, 1998.

PASETTI, S. R.; GONÇALVES, A.; PADOVANI, C. R. Corrida em água profunda para melhora da aptidão física de mulheres obesas: estudo experimental de grupo único. In: SIMPÓSIO INTERNACIONAL DE CIÊNCIAS DO ESPORTE, 27., São Paulo, 2004. Anais... São Paulo: CELAFISCS, 2004.

PATE, R. et al. Physical activity and public health: a recommendation from the Centers for Disease Control and Prevention and the American College of Sports Medicine. JAMA, v. 273, n.5, p. 402-407. Disponível em: < http://wonder.cdc.gov/wonder/prevguid0>. Acesso em: 15 dez. 2007. 
PELLEGRINI FILHO, A. O desafio é a mudança de prioridade: entrevista. RADIS, v. 45, n. 28, 2006.

SECLÉN-PALACÍN, J.; JACOBY, E. R. Factores sociodemográficos y ambientales asociados com la actividad física deportiva en la población urbana del Peru. Rev Panam Salud Publica, v.14 n. 4 p. 255 264, 2003.

SIGERIST, H. E. The university at the crossroads. New York: Henry Schumann, 1946.

TARLOV, A. Social determinantes of health: the sociobiological translation. In: BLANE, D.; BRUNNER, E.; WILKINSON, R. (Ed.). Health and social organization. London: Routledge, 1996.

WORLD HEALTH ORGANIZATION (WHO). Community empowerment for health and development.

Regional Office for the Eastern Mediterranean, 2003. Disponível em: $<$ http://dosei.who.int/uhtbin/cgisirsi/sBpeU9RHyG/199310025/2/1000>. Acesso em: 06 dez. 2007. WORLD HEALTH ORGANIZATION (WHO). Health promotion and healthy lifestyles: A57/Vr/8. Geneva, 2004. 


\section{Ana Paula Martins Vicentin}

Faculdade de Educação Física - UNICAMP

\section{Aguinaldo Gonçalves}

\section{Faculdade de Educação Física - UNICAMP}

\section{Referência do artigo}

\section{ABNT}

VICENTIN, A. P. M.; GONÇALVES, A. Saúde coletiva e atividade física: as políticas públicas respondem e incorporam as realidades setoriais. Conexões, v. 7, n. 2, p. 24-37, 2009.

\section{APA}

Vicentin, A. P. M., \& Gonçalves, A. (2009). Saúde coletiva e atividade física: as políticas públicas respondem e incorporam as realidades setoriais. Conexões, 7(2), 24-37.

\section{VANCOUVER}

Vicentin APM, Gonçalves A. Saúde coletiva e atividade física: as políticas públicas respondem e incorporam as realidades setoriais. Conexões, 2009; 7(2): 24-37.

Recebido em: 13/04/2009

Aceito para publicação em: jun./2009 